\title{
Patterning of metallic glasses using polymer templates
}

\author{
Z. Hu, S. Gorumlu, B. Aksak, G. Kumar* \\ Department of Mechanical Engineering, Texas Tech University, Lubbock, Texas 79409, United \\ States
}

\begin{abstract}
We demonstrate patterning of metallic glasses using flexible and reusable polymer templates. The elastic deformation of polymer templates is utilized to pattern features of varying dimensions and oblique angles on planar and non-planar surfaces. This is enabled by low thermoplastic processing temperature of certain metallic glasses and the stability of thermosetting polymers used as the mold making material. The polymer templates are fabricated by standard replica molding of silicon master templates. This provides a scalable method for patterning of metallic glasses which otherwise requires expensive disposable silicon templates.
\end{abstract}

*Coressponding author:

Dr. Golden Kumar

golden.kumar@ttu.edu

Tel: 806-834-8123 
Patterning of materials is an integral component of fabrication process in microelectronics [1], optical devices [2], and surface engineering [3]. There is an overwhelmingly growing demand for patterning smaller features on novel materials along with reduction in cost of scalability. As a result, tremendous progress has been made in advancement of patterning techniques such as lithography [4], nano-imprinting [5, 6], embossing [3, 7], selfassembly [8, 9], LIGA [10, 11], and laser processing [12]. Despite these efforts, patterning of metallic materials is far less advanced compared to polymers and semiconductors. This deficit is related to inherent challenges in metal processing such as high surface energy, oxidation, rapid grain growth, and incompatibility with mechanical embossing. Recent work on thermoplastic molding of metallic glasses (MGs) appears to bridge this processing capability gap between metals and polymers [13-17]. Pattern features in the range of sub-100 nm have been demonstrated by thermoplastic embossing of MGs [16, 18-20]. After embossing, the MGs can be crystallized by thermal annealing if patterns in crystalline metals are desired [21]. Though thermoplastic shaping of MGs exhibits a great potential for scientific studies [19, 22-25], the template cost poses a major hurdle for large-scale implementation. Typically, silicon templates prepared by lithography are used because of their precision and rigidity [15, 17]. MGs cannot be released from silicon templates without etching because of their thermal expansion mismatch and scalloping roughness of silicon templates. This limits template usage to a single molding operation even for low-aspect-ratio features without undercuts. Additionally, the employment of rigid templates limits the patterning application to planar surfaces. To overcome these issues, we explore the application of polymer templates for patterning of MGs with low glass transition temperatures. The polymer templates offer several advantages such as: inexpensive fabrication by replica molding of master template, ease in demolding, reusability, and conformation to non- 
planar shapes [26-28]. Furthermore, elastic deformation (stretching, compression or bending) of polymer templates can be used to vary the size and orientation of features without the need of a new template [29]. The key requirement for using polymer templates is their stability at thermoplastic molding temperatures $\left(\sim 150-450^{\circ} \mathrm{C}\right)$ of MGs. There is a wide range of thermosetting polymers which are stable up to $300^{\circ} \mathrm{C}$. In the present study, we used PDMS (Polydimethylsiloxane) as a template for Pt-based $\left(\mathrm{Pt}_{57.3} \mathrm{Cu}_{14.6} \mathrm{Ni}_{5.3} \mathrm{P}_{22.8}\right) \mathrm{MG}$, which has the glass transition temperature $\left(T_{\mathrm{g}}\right)$ of $230^{\circ} \mathrm{C}$ [30]. The technique described here can be applied to other combinations of thermosetting polymers and low $T_{\mathrm{g}}$ MGs such as Ce-based [31], Mg-based [32], and Au-based [33]. PDMS (Sylgard 184, Dow Corning) base and curing agent were mixed in 5:1 ratio followed by degasing in a vacuum chamber for $5 \mathrm{~min}$. The degassed PDMS mixture was poured onto the master template and was cured at $70^{\circ} \mathrm{C}$ for $24 \mathrm{~h}$. The thermoplastic embossing experiments were conducted at $270^{\circ} \mathrm{C}$ using a custom-built set-up described elsewhere [21, 34].

Fabrication of polymer templates and their use in patterning of MGs is illustrated in Fig. 1. Lithographically patterned silicon or a thermoplastically patterned MG can be used as the master template. The features of master template are replicated in a cross-linking polymer by standard replica molding process (casting and curing). Once cured, the polymer serves as an inexpensive template for the subsequent patterning of multiple MGs. An additional MG disk was placed at the unpatterned side of the polymer template to create similar boundary conditions on the both sides of the template. The SEM (Scanning Electron Microscopy) images of the silicon master template (Fig. 1b), the polymer replica (Fig. 1c), and the subsequently patterned MG (Fig. 1d) demonstrate the feasibility of the proposed fabrication approach. The master template consists of square shaped micro-pillars $(25 \times 25 \mu \mathrm{m})$ fabricated by photolithography and DRIE (Deep-Reactive-Ion-Etching). The PDMS precisely replicated the features of the silicon master 
template, resulting in formation of square shaped holes. The textured PDMS could be used multiple times to pattern MG samples because of nondestructive demolding facilitated by the flexibility of the template. As shown in Fig. 1d, the size, the shape, and the spacing of features are highly uniform in Pt-based MG patterned using the PDMS template. There is a clear increase in the size of the pattern features in the MG compared to the master (and the polymer) template because of the elastic deformation of the polymer template during compression molding. This dimensional change in pattern features can be predicted by using finite element modelling based on the loading response of the polymer [26]. The master template can be designed to account for the elastic deformation of polymers for a desirable final pattern in MGs. Alternatively, polymer templates with higher stiffness can be used to minimize the variation in pattern dimensions. Besides PDMS, many other thermosetting polymers exhibit suitable properties for applications as templates for patterning of MGs. The properties of template materials that affect the outcome of MG patterning are: thermal stability, Young's modulus, compressive yield strength, and strain limit. These properties for few common thermosetting polymers and their applications as templates are listed in Table 1. It should be noted that the mechanical property data are from room temperature measurements and should be used only for a comparison. For quantitative modelling of template deformation during embossing the mechanical response of polymers at the embossing temperature should be measured. The maximum service temperature of common thermosetting polymers suggests their applicability to a range of MGs such as Ce-based, Aubased, Mg-based, La-based, and Pt-based. Further increase in thermal stability of polymers through composite approach may extend their use for other MGs with higher glass transition temperature. 
Thermoplastic patterning of MGs using rigid templates is well parameterized in terms of embossing temperature and pressure [35-37]. However, the lateral dimensions (diameter and spacing) of features are fixed by the rigid template and cannot be varied by processing conditions. The use of flexible templates, however, introduces an additional variable - template deformation- that can be utilized to control the patterning outcome without changing the template. One way to affect the template deformation, and hence the pattern dimensions, is by varying the embossing pressure. Figure 2 shows the SEM images of Pt-based MG pressed on the same PDMS template under $150 \mathrm{~N}$ (Fig. 2a), $200 \mathrm{~N}$ (Fig. 2b), and $300 \mathrm{~N}$ (Fig. 2c). Microstructural characterization of these samples reveals a systematic variation in size and spacing of pattern features with applied load. Individual pillar size (d1) increased from $36.3 \mu \mathrm{m}$ (@150 N) to $42.6 \mu \mathrm{m}(@ 200 \mathrm{~N})$, and ultimately to $48.7 \mu \mathrm{m}(@ 300 \mathrm{~N})$. The edge-to-edge spacing (d2) decreased from $64.3 \mu \mathrm{m}(@ 150 \mathrm{~N}$ ) to $63.6 \mu \mathrm{m}$ (@200 N), and finally to $61.8 \mu \mathrm{m}$ (@300 N).

The load-dependent dimensional change in MG features stems from the template's incompressible nature which inversely converts the large vertical strain caused by compressive stress into lateral expansion. This enables patterning of MGs with features of different size and spacing using a single template, which significantly lowers the processing cost compared to the conventional approach that relies on customized silicon templates. In addition, the MG features molded using polymer templates displayed smooth surface whereas silicon templates often transfer scalloping roughness to the MG surface [38].

Besides the variation in feature size under uniform deformation, the flexible templates can also be subjected to non-uniform deformation for patterning of inclined features on MGs. Figure 3 shows an example of one such scenario where the deformation of PDMS template was 
altered by mismatching the boundary conditions at two surfaces of the PDMS. Use of only one MG disk during embossing results in asymmetric deformation of PDMS template (Fig. 3a). Top part of PDMS template expanded more due to the presence of viscous MG whereas the lower part of the PDMS template remained pinned on a steel heating plate. Figures $3 b$ and $3 c$ show the SEM images of Pt-based MG embossed on PDMS template using one disk approach under 200 $\mathrm{N}$ and $250 \mathrm{~N}$, respectively. In both cases, the MG pillars are inclined towards the center of the disk. The formation of tilted MG pillars is a consequence of uneven lateral expansion of PDMS mold through its cross-section during compressive loading. PDMS is known to have a large friction coefficient on steel surface [39], which limits the lateral expansion of the bottom surface of PDMS template compared to the upper surface. As a result of frictional disparity at two surfaces, the PDMS template deformed into an inverse trapezoidal shape resulting in formation of tilted cylindrical pores. Subsequent filling of tilted PDMS pores results in the formation of tilted MG pillars. The tilting angle can be controlled by varying the load or by changing the friction between the template and the heating plate. The fabrication of inclined pillars by frictional mismatching of flexible template is simple compared to the existing fabrication techniques that require either electron-beam irradiation [40] or directional resist exposure [27, 41] to make angled structures.

Patterning of non-planar surfaces is inherently challenging by using rigid templates which cannot make conformal contact with a non-planar surface. In contrast, polymer templates offer a unique advantage for non-planar patterning as demonstrated in Fig. 4. A Pt-based MG disk was embossed between a metal block with V-shaped cavity and a flexible PDMS template with micro-holes (Fig. 4a). The MG disk takes the shape of the cavity while replicating the features from the flexible template. The Pt-based MG disk was concurrently patterned and 
deformed into a V-shaped hollow structure. The approach can also be applied for patterning of pre-existing solid MG structures by embossing a thin flexible template around them (Fig. 4b). A V-shaped solid Pt-MG was embossed against a thin PDMS template conformably placed in a machined cavity (Fig. 4b). The MG structure retained V-shaped geometry while replicating the features of the PDMS template. Use of flexible templates in non-planar patterning is particularly advantageous compared to out-of-plane blow molding which is limited to hollow MG geometries [42]. This may be potentially beneficial for roll-to-roll imprinting which requires cylindrical patterned templates.

In summary, we have shown the proof-of-concept for using inexpensive and reusable polymer templates for patterning of MGs. Polymer templates are particularly suitable for patterning of protruded features on MGs due to the stability of polymers pores compared to the pillars during compressive loading. Pattern features of varying dimensions and orientation were fabricated by utilizing the elastic deformation of the template. The extent of template deformation can also be varied by choosing polymers with different elastic moduli (Table 1). Soft polymers such as silicone rubber and PDMS are beneficial for larger variation in feature size and patterning of non-planar surfaces. The patterning of features with higher dimensional accuracy and aspect-ratio can be achieved by using polymers with higher Young's modulus (e.g. SU8 and epoxy resin). The improvement in precision through use of stiff polymer templates however comes at an expense of their reusability. For example, SU8 template will produce high fidelity MG patterns but its rigidity will not allow nondestructive demolding. Nonetheless, the fabrication of polymer templates (disposable or reusable) is still less expensive compared to silicon templates. Alternative strategies such as constraining the templates or using thinner templates can also be devised to optimize the patterning outcome. 
Table 1: Maximum service temperature and mechanical properties of some common thermosetting polymers for template applications.

\begin{tabular}{|c|c|c|c|c|c|}
\hline Template & $\begin{array}{c}\text { Maximum } \\
\text { service } \\
\text { temperature } \\
\left({ }^{\circ} \mathrm{C}\right)\end{array}$ & $\begin{array}{r}\text { Young's } \\
\text { modulus } \\
(\mathrm{MPa})\end{array}$ & $\begin{array}{c}\text { Compressive yield } \\
\text { strength } \\
\text { (MPa) }\end{array}$ & $\begin{array}{c}\text { Elongation } \\
\text { limit } \\
(\%)\end{array}$ & $\begin{array}{c}\text { Applications } \\
\text { as a template } \\
\text { material }\end{array}$ \\
\hline $\begin{array}{l}\text { Silicone } \\
\text { rubber }\end{array}$ & $<250$ & $\sim 1$ & $<20$ & $90-1000$ & $\begin{array}{c}\text { Feature size } \\
\text { variation and } \\
\text { non-planar } \\
\text { patterning }\end{array}$ \\
\hline PDMS & $<280$ & $5-10$ & $\begin{array}{c}\text { 30-50 (ultimate } \\
\text { strength) }\end{array}$ & 160 & $\begin{array}{c}\text { Feature size } \\
\text { variation and } \\
\text { non-planar } \\
\text { patterning }\end{array}$ \\
\hline Polyurethane & $<250$ & $70-1000$ & $30-100$ & $\sim 800$ & $\begin{array}{l}\text { Medium- } \\
\text { aspect-ratio } \\
\text { features }\end{array}$ \\
\hline SU-8 & $<250$ & $\begin{array}{l}2000- \\
4000\end{array}$ & $50-75$ & $4-6$ & $\begin{array}{l}\text { Precise and } \\
\text { high-aspect- } \\
\text { ratio features }\end{array}$ \\
\hline Epoxy resin & $<300$ & $>2000$ & $\sim 80$ & $5-10$ & $\begin{array}{l}\text { Precise and } \\
\text { high-aspect- } \\
\text { ratio features }\end{array}$ \\
\hline
\end{tabular}

\section{Acknowledgement}

G.K. acknowledges financial support from NSF through CMMI-1266277. The SEM characterization was conducted using Hitachi S-4300 acquired through NSF Major Research Instrumentation Program Award \#0421032. 


\section{References}

[1]. W. Lu and C. M. Lieber, Nat. Mater. 6 (2007) 841.

[2]. S. Lal, S. Link and N. J. Halas, Nat. Photon. 1 (2007) 641.

[3]. Y. N. Xia, J. A. Rogers, K. E. Paul and G. M. Whitesides, Chem. Rev. 99 (1999) 1823.

[4]. Y. Xia and G. M. Whitesides, Langmuir 13 (1997) 2059.

[5]. $\quad$ S. Y. Chou, P. R. Krauss and P. J. Renstrom, Science 272 (1996) 85.

[6]. L. J. Guo, Adv. Mater. 19 (2007) 495.

[7]. M. Heckele and W. K. Schomburg, J. Micromech. Microeng. 14 (2004) R1-R14.

[8]. J. Aizenberg, A. J. Black and G. M. Whitesides, Nature 398 (1999) 495.

[9]. G. M. Whitesides and B. Grzybowski, Science 295 (2002) 2418.

[10]. E. W. Becker, W. Ehrfeld, P. Hagmann, A. Maner and D. Münchmeyer, Microelec. Eng. 4 (1986) 35.

[11]. C. K. Malek and V. Saile, Microelec. J. 35 (2004) 131.

[12]. H. Gao, Y. Hu, Y. Xuan, J. Li, Y. Yang, R. V. Martinez, C. Li, J. Luo, M. Qi and G. J. Cheng, Science 346 (2014) 1352.

[13]. Y. Saotome, K. Imai, S. Shioda, S. Shimizu, T. Zhang and A. Inoue, Intermetallics 10 (2002) 1241.

[14]. Y. Saotome, K. Itoh, T. Zhang and A. Inoue, Scripta Mater. 44 (2001) 1541.

[15]. J. Schroers, Jom-Us 57 (2005) 35.

[16]. G. Kumar, H. X. Tang and J. Schroers, Nature 457 (2009) 868.

[17]. G. Kumar, A. Desai and J. Schroers, Adv. Mater. 23 (2011) 461.

[18]. J. P. Chu, H. Wijaya, C. W. Wu, T. R. Tsai, C. S. Wei, T. G. Nieh and J. Wadsworth, Appl. Phys. Lett. 90 (2007) 034101.

[19]. J. Ma, X. Y. Zhang, D. P. Wang, D. Q. Zhao, D. W. Ding, K. Liu and W. H. Wang, Appl. Phys. Lett. 104 (2014) 173701.

[20]. P. Sharma, N. Kaushik, H. Kimura, Y. Saotome and A. Inoue, Nanotechnology 18 (2007) 035302.

[21]. M. Hasan, J. Schroers and G. Kumar, Nano. Lett. 15 (2015) 963.

[22]. N. Li, T. Xia, L. Heng and L. Liu, Appl. Phys. Lett. 102 (2013) 251603.

[23]. M. Kanik, P. Bordeenithikasem, G. Kumar, E. Kinser and J. Schroers, Appl. Phys. Lett. 105 (2014) 131911.

[24]. R. C. Sekol, G. Kumar, M. Carmo, F. Gittleson, N. Hardesty-Dyck, S. Mukherjee, J. Schroers and A. D. Taylor, Small 9 (2013) 2081.

[25]. J. Schroers, G. Kumar, T. Hodges, S. Chan and T. Kyriakides, JOM 61 (2009) 21.

[26]. Y. N. Xia, E. Kim, X. M. Zhao, J. A. Rogers, M. Prentiss and G. M. Whitesides, Science 273 (1996) 347.

[27]. B. Aksak, M. P. Murphy and M. Sitti, Langmuir 23 (2007) 3322.

[28]. Y. N. Xia, J. J. McClelland, R. Gupta, D. Qin, X. M. Zhao, L. L. Sohn, R. J. Celotta and G. M. Whitesides, Adv. Mater. 9 (1997) 147.

[29]. Y. N. Xia and G. M. Whitesides, Adv. Mater. 7 (1995) 471.

[30]. B. A. Legg, J. Schroers and R. Busch, Acta. Mater. 55 (2007) 1109.

[31]. B. Zhang, D. Q. Zhao, M. X. Pan, W. H. Wang and A. L. Greer, Phys. Rev. Lett. 94 (2005) 205502.

[32]. N. H. Pryds, Mat. Sci. Eng. A 375 (2004) 186. 
[33]. J. Schroers, B. Lohwongwatana, W. L. Johnson and A. Peker, Appl. Phys. Lett. 87 (2005) 061912.

[34]. L. Liu, M. Hasan and G. Kumar, Nanoscale 6 (2014) 2027.

[35]. D. L. Henann, V. Srivastava, H. K. Taylor, M. R. Hale, D. E. Hardt and L. Anand, J. Micromech. Microeng. 19 (2009) 115030.

[36]. G. Kumar, J. Schroers and J. Blawzdziewicz, Nanotechnology 24 (2013) 105301.

[37]. J. Ma, L. S. Huo, D. Q. Zhao and W. H. Wang, J. Appl. Phys. 113 (2013) 104505.

[38]. G. Kumar, J. Schroers, Appl. Phys. Lett. 92 (2008) 031901.

[39]. I. D. Johnston, D. K. McCluskey, C. K. L. Tan and M. C. Tracey, J. Micromech. Microeng. 24 (2014) 035017.

[40]. M.-W. Moon, T.-G. Cha, K.-R. Lee, A. Vaziri and H.-Y. Kim, Soft Matter. 6 (2010) 3924.

[41]. H. E. Jeong, J. K. Lee, H. N. Kim, S. H. Moon and K. Y. Suh, P. Natl. Acad. Sci. USA 106 (2009) 5639.

[42]. J. Schroers, T. M. Hodges, G. Kumar, H. Raman, A. J. Barnes, P. Quoc and T. A. Waniuk, Mater. Today. 14 (2011) 14. 


\section{Figure captions}

Fig. 1: Fabrication of textured polymer and their use as templates for thermoplastic patterning of MGs. Schematic illustration of the processing scheme (a), an example of silicon master template with micro-pillars (b) and its tilted view (inset), a PDMS template with micro-holes prepared by replica molding (c), and a Pt-based MG surface patterned by using the PDMS as a template (d). Two MG disks, one on each side, are pressed to establish similar boundary conditions on the top and the bottom surfaces of the PDMS template.

Fig. 2: SEM images of Pt-based MG embossed on PDMS template at $150 \mathrm{~N}$ (a), $200 \mathrm{~N}$ (b), and $300 \mathrm{~N}$ (c). The insets show images taken after tilting the samples at $90^{\circ}$. The pillar size (d1) increases whilst the edge-to-edge spacing (d2) decreases with increasing embossing load.

Fig. 3: Schematic illustration for patterning of inclined features using non-uniform deformation of an elastomeric template (a) and the SEM images of Pt-based MG patterned with inclined pillars prepared at $200 \mathrm{~N}$ (b) and $250 \mathrm{~N}$ (c). The pillars are tilted towards the center of the sample.

Fig. 4: Patterning of hollow (a) and solid (b) non-planar MG structures using flexible polymer templates. For hollow structures, a thick polymer template is used to reshape and pattern the MG simultaneously (a). Solid MG structures can be patterned by embossing a thin polymer template that makes conformal contact with the non-planar surface. The proof-of-concept is demonstrated by patterning V-shaped hollow and solid geometries of Pt-based MG using a PDMS template with micro-holes. 

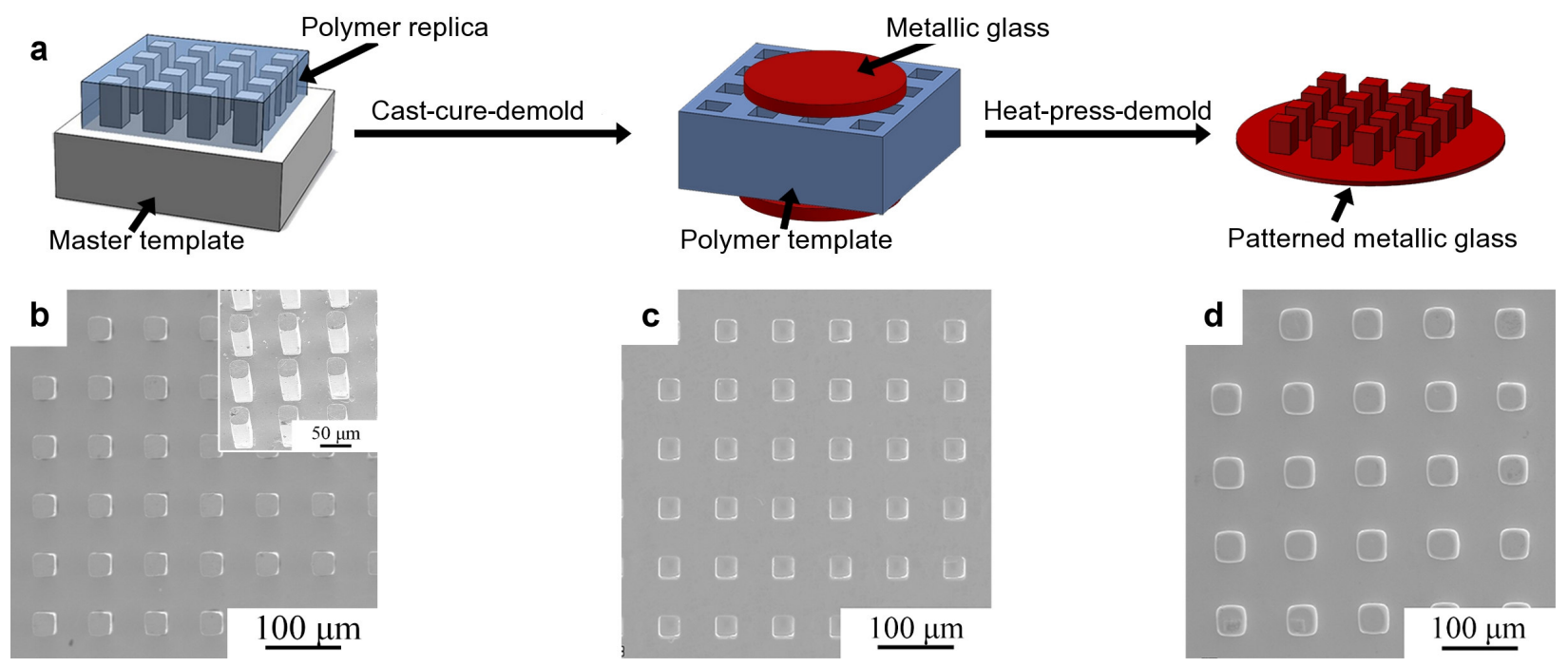

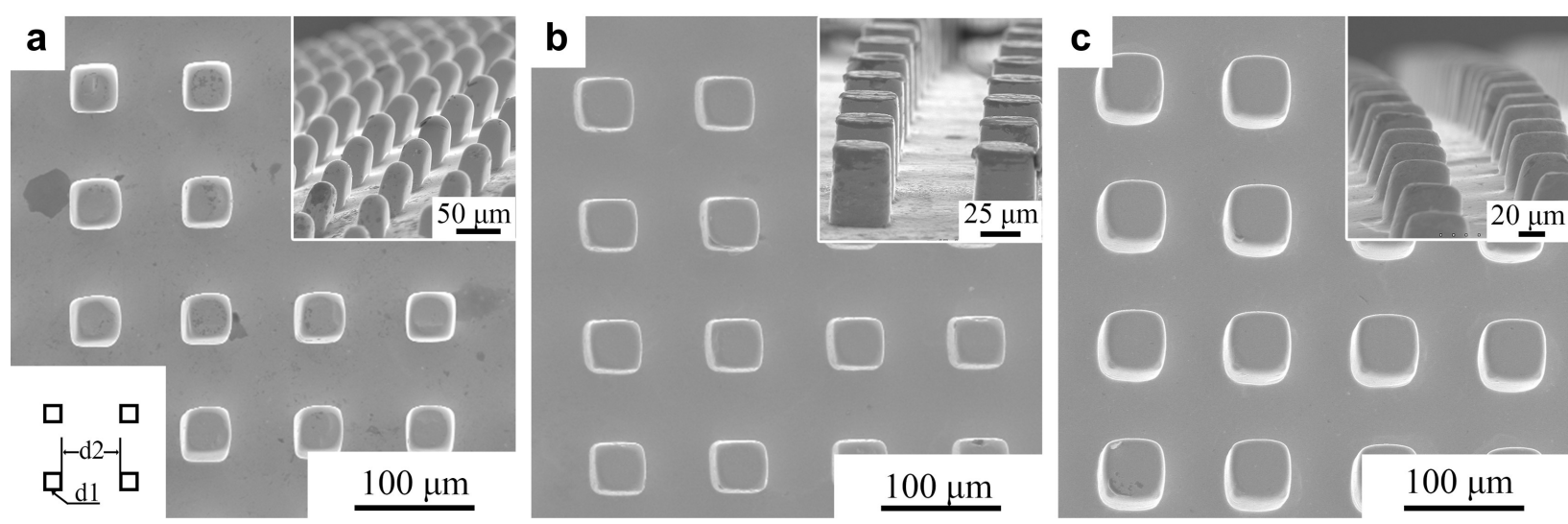
a

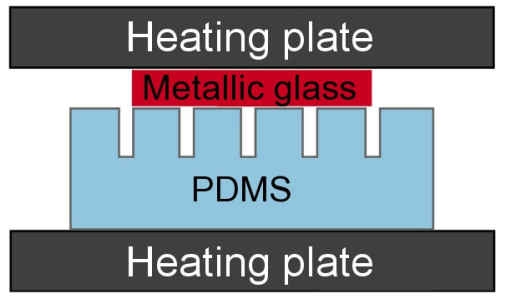

b

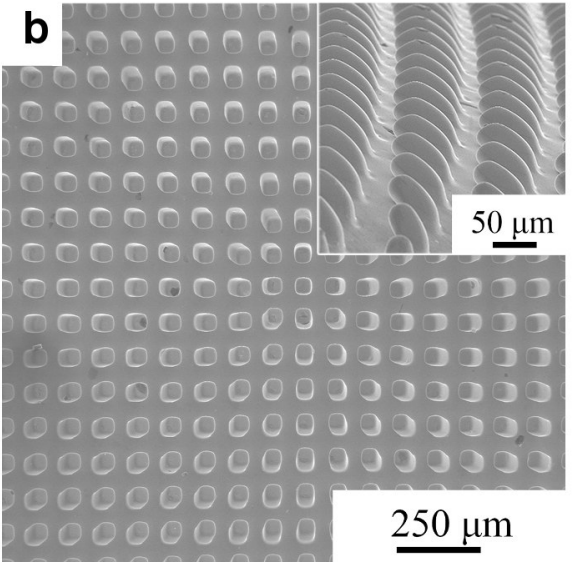

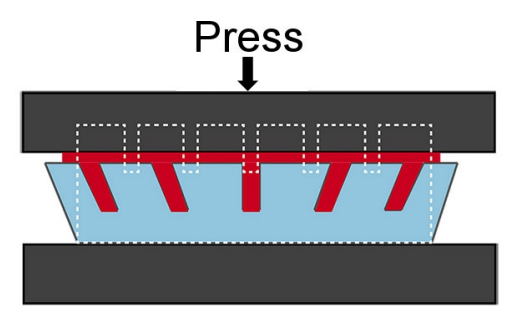

c

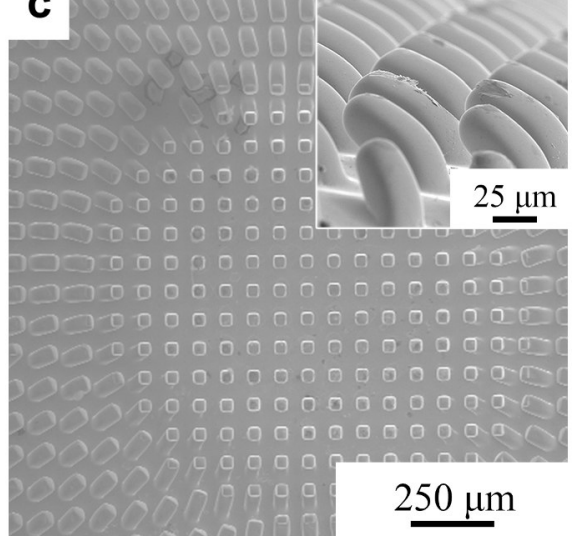


a

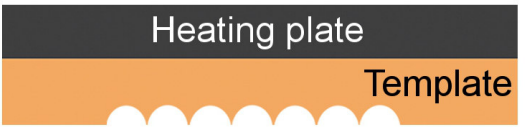

Metallic glass

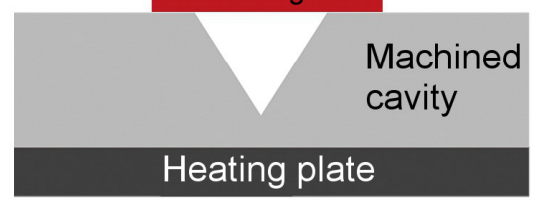

b

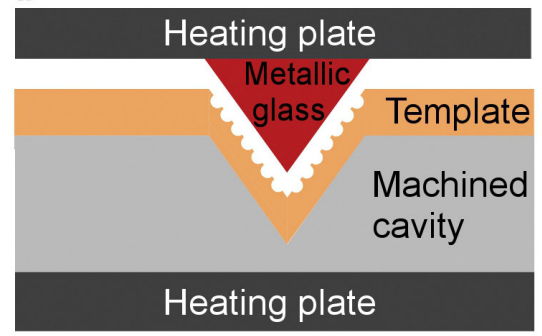

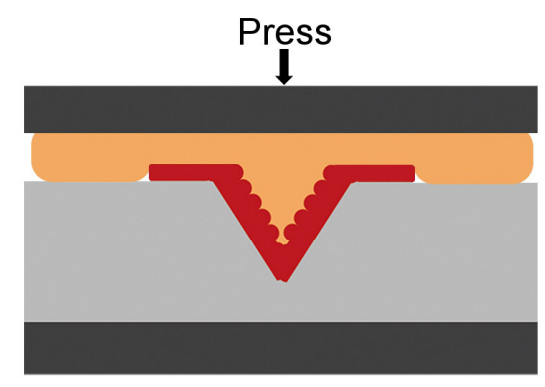

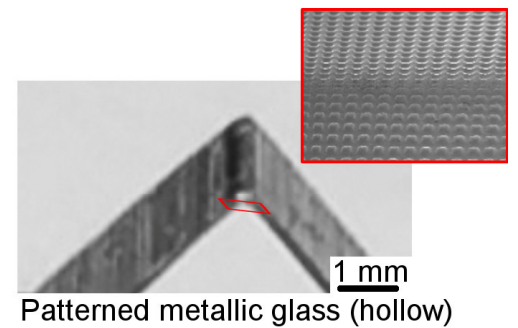

\section{Press}

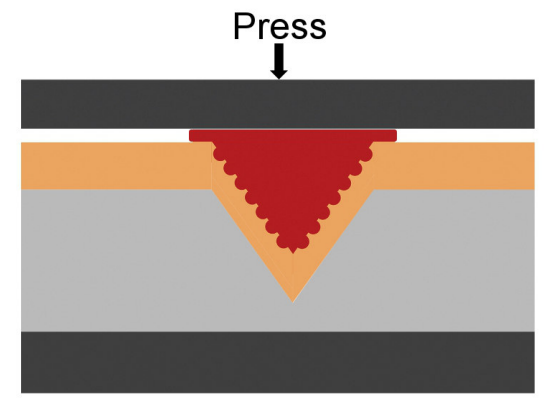

Patterned metallic glass (hollow)

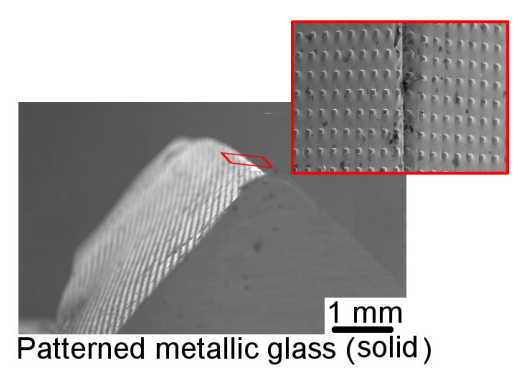




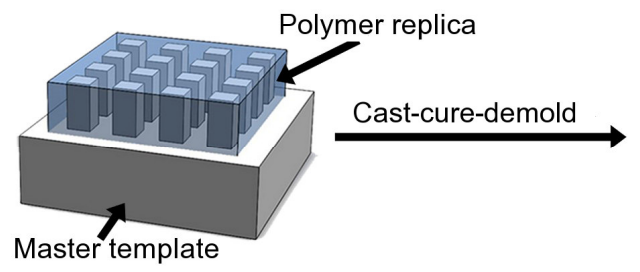

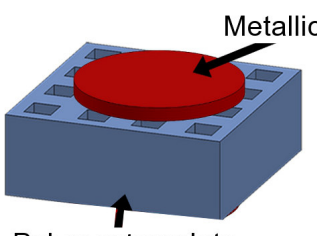

Polymer template

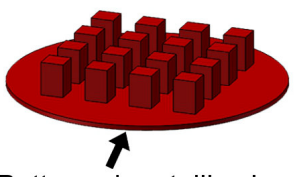

Patterned metallic glass 